\title{
La Diputación Provincial de Sevilla y el advenimiento de la dictadura de Primo de Rivera (1923-24)
}

\section{JULIO PONCE ALBERCA}

Sabido es que, desde su orígen, las Diputaciones provinciales buscaron desenvolverse en el complejo -y a veces confuso- espacio de la función pública española dentro del segmento intermedio entre la Administración Central y los Municipios. Durante el siglo XIX numerosos ordenamientos intentaron fijar el papel de las Diputaciones Provinciales según el matíz de la orientación política -moderada o progresista-del grupo que ostentara el Poder. Más tarde, superada la etapa revolucionaria y una vez consolidado el modelo liberal, ambas opciones políticas tendieron a converger en la consideración de las competencias que habrían de jugar los organismos provinciales. De esa convergencia se decantaron las características básicas que definirían y distinguirían a las Diputaciones desde finales del siglo XIX: la fuerte dependencia con respecto al Poder central (vía Gobierno Civil) y la reducción de sus competencias en materia administrativa que, o bien serían meras delegaciones del Gobierno en el entorno provincial, o bien se circunscribirían a aquellas parcelas de gestión que el Estado no llegaba a cubrir. Estos caracteres quedaron sujetos al fuerte revestimiento político con el que se les dotó en su calidad de organismo de control de Ayuntamientos y asesor vinculado al Goberna dor Civil.

Evidentemente, esa variedad de funciones político-administrativas no clarificó en ábsoluto los límites de las heterogéneas capacidades de las Diputaciones sino que, por el contrario, provocó una peligrosa mezcla de ambos ámbitos en la provincia -el político y el de la gestióndando lugar a desajustes negativos en el desempeño de las misiones que la Ley les encomendaba. Como órgano político, las Diputaciones se encontraron, pues, estrechamente subordinadas al Poder central a través de la figura del Gobernador Civil ocupándose a su vez, como entidad superior a los Ayuntamientos, de intervenir en la vida política y económica local mediante sus resoluciones en materias clave como elecciones, quintas, impuestos, etc. Se incardinaba así a las Diputaciones en el meo- 
llo del juego de la política caciquil gracias a su emplazamiento estratégico entre el Gobierno Civil y los Ayuntamientos y merced, también, al control en la provincia de órganos trascendentes como la Junta Electoral. Esa implicación de las Diputaciones en funciones políticas desvirtuó desde un primer momento el desarrollo de sus competencias administrativas que, aun siendo múltiples, se vieron reducidas al ineficaz sustento de la beneficencia provincial y a alguna tímida actuación en las obras públicas de la provincia, dentro de un clima indudable de penuria hacendística. Estos caracteres fueron consolidados a partir de la prómulgación de la Ley provincial de agosto de 1882 , ordenamiento determinante para la comprensión del desenvolvimiento de las Diputaciones durante el primer cuarto del siglo XX.

Dicho lo anterior, no extraña que la Ley de 1882 -afianzadora en la práctica del caciquismo provincial- fuera objeto de duras críticas por parte de aquellos que ponían en cuestión el funcionamiento del sistema político nacional. Estas críticas coetáneas denunciaron la misión decisiva que jugaban las Diputaciones a la hora de preparar unas elecciones, el escaso margen de independencia que gozaban y la presión abusiva que ejercían los organismos provinciales sobre las haciendas locales a la hora de exigir el contingente: pieza clave, dicho sea de paso, de la financiación provincial exigida a los Ayuntamientos bajo su control. Los críticos no solo señalaron con irritación la escasa eficacia administrativa de los organismos provinciales, sino que incluso llegaron a cuestionar explícitamente hasta la oportunidad y validez de su propia existencia. No obstante, es obligado reseñar que, dentro de este criticismo, se encontraba una corriente muy significativa que propugnaba como solución del sistema la reforma de la Administración Provincial y Local como paso previo en el proceso de regeneración nacional. Así, como afirmaba el que fuera Director General de Obras Públicas en uno de los gabinetes Silvela, Pablo Alzola:

*... el remedio está precisamente en España en sentido contrario,

en vigorizar las extremidades evitando la atrofia del corazón ${ }^{1}$.

Esa vigorización se entendía vinculada a una mayor autonomía para las provincias en sentido descentralizador. En esta sensibilización general del problema -alcanzando hasta las más altas instancias- hemos de inscribir los esfuerzos reformadores de la administración provincial que, entre otros, protagonizarían hombres como Silvela, Maura, Canalejas o Cambó.

Tras lo expuesto se entiende, pues, que en la reorganización de las Diputaciones se centrara una de las claves de la regeneración del sistema canovista. Así, al menos, lo entendió Maura que, bajo los postulados de su

(1) Alzola y Minondo, P.: Historia de las obras puiblicas en España, (Madrid, Turner, 1979), p.453. 
revolución desde arriba, elaboró en 1907 un proyecto de reforma de la Administración Local que, en lo referente a Diputaciones, retocaba parcialmente la Ley de 1882 reservando una mayor descentralización y autonomía para estos organismos permitiéndoles, de esta forma, una presumible desvinculación del encasillado caciquesco. Al mismo tiempo, el proyecto establecía un reforzamiento de las haciendas provinciales llegando, en último extremo, a observar -con sus reservas correspondientes- la posibilidad de que las Diputaciones formasen mancomunidades interprovinciales..

Aparte valoraciones de estos intentos reformistas -lo que queda fuera de la pretensión de estas líneas generales- lo cierto es que dichos proyectos permanecieron en el plano de la letra escrita en fuerte contraste con la realidad objetiva del momento. $Y$ es que los mismos partidos del turno, que reconocían la necesidad de la reforma administrativa, eran conscientes de que su supervivencia política dependía del control electoral y, para continuar ejerciendo éste, se mostraban reacios a una transformación de las Diputaciones que alterase peligrosamente su status quo. Por ello, mientras el Poder derivase de la manipulación de los sufragios, los proyectos de reforma no tendrían aplicación práctica. De ahí se explica por qué la Dictadura de Primo de Rivera, desvinculada por su propia naturaleza política del proceso electoral, fue capaz de poner al menos en marcha una propuesta de reforma de la Administración Local, recogiendo las aspiraciones regeneracionistas filtradas en los postulados del conservadurismo español del momento: el Estatuto Municipal de 1924 y el Estatuto Provincial de 1925.

Situado en la encrucijada que significó el año 1923, el tema de las Diputaciones volvió a cobrar tal protagonismo que se hace hoy de sumo interés el análisis del 'comportamiento de las Diputaciones Provinciales bajo los efectos de los últimos meses del sistema de la Restauración y los primeros de la Dictadura de Primo de Rivera. En este caso y a través del análisis del comportamiento político de la Diputación sevillana desde junio de 1923 (últimas elecciones provinciales) hasta enero de 1924 (nombramiento de las primeras corporaciones provinciales bajo el Directorio), intentaremos responder a varios interrogantes entre los que destacamos los siguientes:

$1^{2}$. Existieron realmente en el ámbito provincial sevillano esfuerzos de purificación democratizadores del sistema que se vieron súbitamente interrumpidos por la irrupción de la Dictadura?

(2) González Casanovas, J. A.: Las Diputaciones provinciales en España. Historia política de las Diputaciones desde 1812 basta 1985 (Madrid, Mancomunidad General de Diputaciones de Régimen Común,1986), pp.68-70. 
$2^{2}$. .Se produjeron cambios sustanciales en el comportamiento de la Diputación Provincial de Sevilla tras el 13 de septiembre de 1923? Y si los hubo, ¿en qué consistieron?

$3^{\circ}$. ¿Se pueden establecer diferencias esenciales entre la primera Corporación provincial nombrada por la Dictadura en enero de 1924 respecto de las de la vieja política?

Veamos esos tres puntos reflexivamente.

\section{Las elecciones provinciales de junio de 1923}

Tras la celebración de las últimas elecciones generales en el mes de abril de 1923, fueron convocadas para el 10 de junio las elecciones provinciales. Estas representaban la culminación del proceso de renovación del poder político en todos los niveles iniciado desde la constitución del Gobierno liberal de García Prieto en diciembre de 1922. Si las elecciones generales de abril -en consonancia con el viciado mecanismo electoral de entonces- dieron la victoria a la candidatura gubernamental ${ }^{3}$, quedaba por dilucidar el resultado de las provinciales que tenían por objeto la elección parcial de los cargos de las Corporaciones de acuerdo con los artículos 44 y 57 de la Ley provincial vigente.

Para estas elecciones, trece eran las vacantes a cubrir en la Diputación distribuídas entre los distritos primero y segundo de la capital (cuatro diputados a elegir en cada una), el distrito de Osuna-Morón que elegía a otros cuatro y el distrito de Utrera-Marchena que ofrecía una vacante por fallecimiento del diputado que la ocupaba: el liberal albista Rafael Romero Gutiérrez.

De acuerdo con las prácticas electorales acostumbradas, el Gobierno pactó con la izquierda liberal para repartirse los cargos vacantes en la Diputación. Un primer pacto concedió ocho diputados para los albistas y cinco para los conservadores, pero no fue aceptado por éstos últimos. Por ello, se procedió a un nuevo compromiso que cedía cuatro vacantes para los albistas, dos para la Unión Comercial, una para los garciprietistas y otra quedaba reservada al Gobierno permaneciendo inalterables los cinco diputados prometidos a los conservadores. Este nuevo pacto reflejaba- el acuerdo tácito al que habían llegado los conservadores con la Unión Comercial y con el garciprietismo frente a los liberales albistas que, aunque en un primer momento protestaron por la repartición, acabaron aceptándola.

(3) Sevilla, en estas elecciones no fue una excepción. De los cinco escaños a elegir por la capital dos fueron a parar a manos liberales, consiguiendo cinco de los ocho diputados a elegir por la provincia. Vid.: Martínez Cuadrado, M.: Elecciones y partidos polúticos en España (1868-1931) (Madrid, Taurus, 1969), cuadro estadístico número 26, p.995. 
El dia 4 se reunió la Junta Provincial del Censo en la Diputación con el objeto de proclamar candidatos y, en su caso, aplicar el socorrido artículo 29 de la Ley Electoral en el mayor número de distritos. Fue así como la mayor parte de las vacantes no necesitaron de elección para ser cubiertas. Los diputados nombrados por el mencionado artículo fueron ${ }^{4}$ :

Distrito 1 (capital)

Manuel Sarasúa Barandiarán (conservador)

Jose María Piñar y Pickman (conservador)

Luis Ruiz de Rebolledo (albista)

Tadeo Soler Navarro (Unión Comercial)

Distrito Osuna-Morón

Jose María Tassara González (conservador)

Aniceto de la Puerta Govantes (conservador)

Eduardo Fierro Izquierdo (albista)

Pedro Pérez Rico (albista)

Distrito Utrera-Marchena

Francisco Molina y Arias de Saavedra (romanonista)

De esta forma, tan sólo en uno de los distritos de la capital -el segundo- se entablaría la lucha electoral al haber mayor número de candidatos que de vacantes. Retirada la candidatura del albista Díaz Hidalgo, los aspirantes a los cargos provinciales por este distrito fueron el conservador Antonio Filpo; el representante de la Unión Comercial, García Junco; el garciprietista Morales Roldán; el republicano Juan Revilla; y el representante de la Liga Católica, José Monge y Bernal.

Tras lo expuesto, se comprueba la fortaleza que presentaban los conservadores en la Diputación sevillana de estas fechas. Una fortaleza que se vio consolidada en estas elecciones en virtud de su pacto con la Unión Comercial y con los garciprietistas, frente a los albistas aislados en la Corporación Provincial. Mientras, los grandes perjudicados del sistema seguían siendo la mayor parte de los partidos externos al turno que no se avenían a componendas con los partidos tradicionales de la vieja política. Este es el caso de los republicanos, que a duras penas lograron integrar candidatura evitando el artículo 29, o el de la Liga Católica que presentó a su candidato ccn el único apoyo de los mauristas.

El segundo distrito, en el que se iban a celebrar las elecciones, estaba compuesto por los distritos cuarto, quinto, sexto, séptimo y octavo de la capital (es decir San Vicente y la Magdalena) más doce pueblos de la circunscripción, a saber: Alcalá del Río, La Algaba, Bormujos, Burguillos, Camas, Castilblanco, Castilleja de Guzmán, El Garrobo, Guillena, Lạ Rinco-

(4) EL CORREO DE ANDALUCIA, 5 de junio de 1923. 
nada, Santiponce y Valencina. Con la inclusión de estos pueblos, se corregía la orientación del voto urbano mediante la añadidura de los sufragios controlados por los caciques de las localidades de la circunscripción.

Como en otras muchas ocasiones, estas elecciones provinciales se distinguieron por el desinterés del electorado y por la manipulación del voto. La prensa se hizo eco de esta desidia electoral: "Ayer dicen comenta El Liberal- que hubo elecciones en Sevilla. Nosotros juraríamos que no. La desanimación fue la nota culminante.5. Por su parte, El Correo de Andalucia recogió una impresión similar sobre unas elecciones que ....transcurrieron en medio de una gran desanimación (...) A nadie importan y nadie vota. Y añadía: *Hubo pucherazos a granel en Sevilla y en los pueblos. No faltó alguna compra de votos en los distritos donde los jefes electorales de ellos hicieron cuestión de amor propio el obtener para su partido gran mayoría sobre el candidato del otro grupo. Así en Triana, donde los conservadores deseaban derrotar grandemente a los republicanos ${ }^{6}$. Tampoco faltaron incidentes como los ocurridos en Bormujos, donde se rompió una urna anulándose la elección; o en Alcalá del Río, localidad en la que :El cuerpo electoral se disponía a otorgar sus votos al liberal Sr. Díaz Hidalgo; pero retirado éste, el cuerpo electoral se desorientó y no constituyó las Mesas*. En Castilblanco fue detenido el interventor republicano León Trejo y no se permitió la entrada de los notarios a los colegios electorales. Tras estas incidencias, en estas poblaciones hubo de procederse a la convocatoria de repetición de las elecciones para el 13 de junio bajo las recomendaciones expresas del Gobernador Civil (Fernández Jiménez) para que ‘bajo ningún pretexto* se detuviera a nadie durante la jornada electoral ${ }^{7}$.

Después de estas vicisitudes, los resultados finales de la elección del segundo distrito arrojaron un resultado favorable a los conservadores y a las fuerzas que habían pactado con ellos (la Unión Comercial y los garciprietistas). Tan solo uno de los puestos de la Corporación fue obtenido por un candidato no perteneciente a los partidos del turno, independiente de cualquier tipo de pacto: el republicano radical Juan Revilla. El candidato católico Jose Monge y Bernal no llegó a ser electo, tal y como podemos observar en el cuadro siguiente:

(5) EL LIBERAL, 12 de junio de 1923.

(6) EL CORREO DE ANDALUCIA, 12 de junio de 1923.

(7) EL LIBERAL, 12 de junio de 1923. 


\section{CANDIDATO}

Antonio Filpo y Rojas (conservador)

Emilio García-Junco y Ruíz (Unión Comercial)

Jose Ignacio Morales Roldán (garciprietista)

Juan Revilla García (republicano)

José Monge y Bernal (Liga Católica)

FUENTE: La Uniōn, 14 junio 1923
VOTOS OBTENIDOS

6622 votos (E).

5466 votos (E).

5447 votos (E).

2935 votos (E).

2633 votos .

\section{Constitución de la nueva Diputación: elementos de persistencia y \\ elementos de cambio}

A comienzos de agosto de 1923 tomaron posesión de sus cargos los nuevos diputados, adquiriendo la nueva Diputación Provincial la siguiente composición ${ }^{8}$ :

Sevilla, primer distrito

Manuel Sarasúa Barandiarán (conservador)

Jose María Piñar y Pickman (conservador)

Luis Ruiz de Rebolledo (albista). Pres.Asamblea Provincial.

Tadeo Soler Navarro (Unión Comercial)

Sevilla, segundo distrito

Antonio Filpo y Rojas (conservador)

Emilio García-Junco y Ruíz (Unión Comercial)

Jose Ignacio Morales Roldán (garciprietista)

Juan Revilla García (republicano)

Distrito Utrera-Marchena

Modesto Cañal y Migolla conservador)

Francisco Molina (liberal romanonista)

Rafael Romero Gutiérrez (liberal albista)

José Gutiérrez Ríos (conservador)

Distrito Cazalla-Sanlúcar

Fernando Arana Martín (liberal albista)

Francisco Rodríguez Pacheco (conservador)

José Delgaço González (liberal albista)

Juan Bellón Jiménez (conservador)

Distrito Osuna-Morón

Cristóbal Puerta Govantes (conservador)

Jose María Tassara González (conservador)

Pedro Pérez Rico (liberal albista)

Eduardo Fierro Izquierdo (lib. albista). Pres.Comisión Perm.

(8) Los distritos de la provincia tomados de: Alvarez Rey, L.: La derecha en Sevilla durante la II República (Sevilla, T.D.I., 1990), f.767. [En prensa]. 


\section{Distrito Ecija-Estepa}

Antonio Huertas Checa (liberal albista)

José León y Manjón, marqués del Valle de la Reina (cons.)

Antonio Lasarte Hidalgo (liberal albista)

Benito Rodríguez Pérez (conservador)

\section{Distrito Carmona-Lora}

Federico Jiménez Jiménez (conservador)

Juan Florindo Moreno (conservador)

Eduardo Solís Rivas (liberal albista)

José Santos y Sánchez Alcobendas (liberal romanonista)

Ahora bien, a pesar de la mayoría conservadora, los cargos de la Presidencia de la Asamblea (Luís Ruíz de Rebolledo) y de la Comisión (Eduardo Fierro Izquierdo) recayeron en albistas respondiendo, al parecer, al acuerdo tácito entre los grupos políticos. Sin embargo, no cabe duda de la existencia de leves rasgos novedosos en la composición de esta Diputación: la inclusión de diputados de la Unión Comercial y de un republicano. ¿Era ello prueba de que algo estaba cambiando o iba a cambiar en el seno de la administración provincial sevillana?

Con esta pregunta no es nuestro propósito entrar en el debate historiográfico en torno a la significación del último gobierno García Prieto ni cuestionar si su presunto intento democratizador fue más o menos sincero frente a la irrupción que representó la Dictadura. En sí, nuestra cuestión no pretende más que averiguar si la entrada de diputados ajenos a los partidos del turno hizo posible la consecución de cambios de importancia en la gestión del organismo provincial y, en su caso, definir el alcance de los mismos. Para ello, comencemos por analizar las características del organismo que se encontraron los nuevos diputados.

Pese a ser mejor que sus homólogas de otras provincias, el funcionamiento de la Diputación Provincial sevillana destacaba hasta el momento por su ineficacia en el apartado administrativo y por su total dedicación al juego político encomendado por el Gobierno central a través del Gobernador Civil. Un repaso a las Actas de la Comisión Provincial nos demuestran hasta que punto es esto cierto. Desde enero a julio de 1923, las actuaciones fundamentales de la Comisión Provincial consistieron en la resolución de los recursos presentados contra la elección de concejales de las últimas elecciones municipales -lo que dió lugar a debates enconados entre liberales y conservadores- ${ }^{9}$ y en la resolución de recursos de alzada formulados contra determinados ceses de personal municipal. Esta administración, politizada y rutinaria, se conjugaba con

(9) Archivo Diputación Provincial de Sevilla (en adelante A.D.P.S.). Actas Comisión Provincial. Sesiones de 5 y 26 de enero de 1923, ff.1-17. 
los seculares problemas de financiación de la Diputación que repercutían, como siempre, sobre las haciendas municipales a partir de la apertura de procedimientos de apremio en razón a la exigencia del contingen$\mathrm{te}^{10}$. Las obras públicas emprendidas por la Diputación fueron numéricamente insignificantes, mientras la beneficencia provincial sevillana era publicamente criticada por la falta de agua en los hospitales ${ }^{11}$ y por el mal trato ofrecido a los enfermos ${ }^{12}$. Y, ciertamente, no debían encontrarse muy lejos de la verdad estas críticas toda vez que la propia Diputación hubo que prohibir el consumo de las aguas del pozo del Hospital de San Lázaro trasladando a los leprosos allí acogidos al Hospital Central ${ }^{13}$.

A la luz de esta situación, no resulta incomprensible que ciertos críticos definieran a la Diputación como *ese organismo fofo, incoloro, inodoro e insípido. ${ }^{14}$. Pero si hubo novedad, ésta consistió en que a partir de las elecciones de junio, con el ingreso de nuevas fuerzas políticas en la Diputación, el propio interior de la corporación provincial comenzó a actuar parcialmente como caja de resonancia de ciertas críticas, clarificándose así las posturas de los distintos grupos políticos que la componían. Evidentemente ese protagonismo crítico no iba a partir ni de liberales ni de conservadores. Así, para el liberal albista Luis Ruíz de Rebolledo, presidente de la Diputación, la herencia recibida de las anteriores corporaciones era en conjunto positiva, opinión que los conservadores -por boca de Sarasúa- rubricaron ${ }^{15}$. Sin embargo, no fue esa la valoración que se hacía de la actuación de la Diputación por parte de los nuevos grupos, que bien pronto demostraron ser los emisores de las notas discordantes en las sesiones. Así, el republicano Juan Revilla al posesionarse de su cargo ya indicó a sus compañeros de corporación que -... la la Diputación fueran todos a hacer labor administrativa y no política*, en curiosa sintonía con una sensibilidad muy acorde respecto a los postulados administrativos del maurismo ${ }^{16}$. Pero aún más explícitos se mostraron los diputados de la Unión Comercial. El diputado Tadeo Soler, en ausencia de su compañero García Junco, tomó la palabra en la misma sesión exponiendo una dura crítica contra la legislación provincial enton-

(10) Ibídem, sesión de 17 de julio de 1923, ff.172-173.

(11) Ibídem, seșión de 29 de mayo de 1923, f.101 r.

(12) Ibídem, sesión de 1 de junio de 1923, f.105 r.

(13) Ibídem, f:105. Es necesario añadir, por otra parte, que la sanidad en Sevilla dejaba mucho que desear según los datos aportados ' por Guichot sobre la incidencia de enfermedades como la fiebre tifoidea, la viruela, la gripe o la tuberculosis pulmonar. Vid: Guichot y Barrera, J.: La provincia de Sevilla estadísticamente considerada (Sevilla, Imp.Alvarez, 1924), pp.46 y ss.

(14) EL CORREO DE ANDALUCIA, 14 de septiembre de 1923.

(15) EL CORREO DE ANDALUCIA, 4 de agosto de 1923.
(16) Ibídem. 
ces vigente, expresando unas intenciones que probablemente inquietaron al propio grupo conservador, responsable de la ascensión de los miembros de la Unión Comercial a la corporación:

-Al aceptar nosotros la designación hecha por la Unión Comercial... renunciamos a todo interés y conveniencia personal, y hasta a nuestras propias convicciones; porque ni el señor García Junco ni yo creemos indispensable, ni siquiera necesaria para la vida de los pueblos, la intervención en sus asuntos de las actuales Diputaciones Provinciales, tal y como estan constituidas, sujetas al poder Central y regidas por una ley anticuada, incompatible con las orientaciones de descentralización y autonomía administrativa que constutuyen hoy la suprema aspiración de los organismos locales, provinciales y regionales de las naciones modernas ${ }^{17}$.

Tras esta andanada inicial, Tadeo manifestó -tranquilizando ánimosque no obstante la Unión Comercial trabajaría desde esa realidad a transformar ....sin perjuicios de ninguna clase, con cariño y lealtad. En esa labor, aseguró que la Unión Comercial sería la defensora :... de los intereses morales y materiales de la provincia, trabajando por el mayor progreso de su cultura, la máxima prosperidad de sus riquezas industrial, comercial y agrícola y por la más fácil y rápida comunicación de sus pueblos entre sí, completando su programa con la intención de mejorar las dependencias de la Casa Diputación "que a primera vista no son buenas" y con el propósito de "... reducir las actuales plantillas al límite, amortizando todas las vacantes que no sean imprescindibles y aumentando el sueldo de los que queden..." porque "...veinte empleados bien retribuídos rinden más y mejor trabajo que cuarenta mal pagados. ${ }^{18}$. A la luz de estas palabras, resultaba evidente que la nueva Corporación prometía una cierta transformación de conductas -al menos internas- en la Diputación sevillana. Y aunque, efectivamente, esa transformación formal se verificó como veremos a continuación, ella no se tradujo en un profundo cambio real en los efectos de la gestión de la Diputación. Al fin y al cabo, ni la Unión Comercial ni el republicano Revilla podían conseguir mucho si tenemos en cuenta su exígüa representación numérica y, sobre todo, la vigencia de la Ley de 1882.

Por tanto, en el corto espacio de tiempo que medió entre la constitución de la nueva Diputación (agosto) y el golpe de Primo de Rivera (septiembre), es detectable una moderada transformación del desarrollo de las sesiones del organismo provincial patentizada en la dinamización de las mismas a cargo de los diputados de la Unión Comercial y del

(17) LA UNION, 3 de agosto de 1923. La cursiva es del autor.

(18) Ibídem. 
diputado republicano. En ese mes largo, estos diputados solicitaron antecedentes al presidente de la Comisión Fierro sobre las deficiencias de la asistencia benéfica "que de público se dice se observan. Este, en postura justificativa, les explicó que las carencias que eran denunciadas *... unas estaban corregidas y otras en vías de corregirse, como lo demuestra la inauguración del lavado mecánico en el Hospital central, efectuada el último sábado, y otras importantes mejoras que en breve plazo se llevarán a la práctica , añadiendo que .... ciertas campañas, no ajenas a un crónico estado de pasión, pueden cesar al hecerse desaparecer los pequeños lunares que ahora se observan", como si el cuestionamiento de la Diputación se centrase sólamente en la gestión benéfica. Fierro prometió "poner de su parte el mejor propósito* en la resolución de este problema augurando que "por cortas que fuesen las consignaciones Ipara beneficencia], si éstas se gastan con tendencia modernista, es seguro que hará desaparecer los anacronismos y deficiencias que se vocinglean, quedando en último extremo...un plan completo de reformas de conjunto, que podrán ser acometidas en diferentes presupuestos ${ }^{19}$.

Evidentemente, aquellas explicaciones que posponían en último extremo un plan integral de reformas no satisfizo al sector crítico de la Corporación. Como se ve, el contraste entre los diputados ajenos a los partidos dinásticos y los pertenecientes a la vieja política se canalizaba a través del debate sobre la beneficencia y, sin llegar a una ruptura formal, daba innegables señales de vida. Para la Unión Comercial era ya pasado el acuerdo que habían tenido que establecer con los conservadores para acceder a la Diputación. La irrupción del golpe primorriverista se encargaría de precipitar la escisión en el seno de la Diputación sevillana.

\section{El golpe de estado de Primo de Rivera y sus efectos sobre la Diputación Provincial de Sevilla}

La noticia del golpe del 13 de septiembre fue recibido de manera expectante y favorab'e por la mayor parte de los sectores de la vida sevillana ${ }^{20}$. Así viene a demostrarlo las manifestaciones de la propia prensa local que, en aquellas jornadas, se adhirió mayoritariamente al pronunciamiento con la excepción aparente del diario La Unión que bajo el título de *iLa Dictadura, no! parecía separarse de la tónica general. En realidad, el órgano portavoz de la Unión Comercial finalizaba su artículo suscribiendo el manifiesto de Primo de Rivera excluyendo la parte dispositiva del mismo y *algunos alardes fanfarrones*, no sin advertir que:

(19) EL LIBERAL, 14 de agosto de 1923. La cursiva es del autor. (20) Braojos, A., Parias, M. y Alvarez, L.: Sevilla en el siglo XX (1868-1950), tomo II, (Sevilla,
Universidad de Sevilla, 1990), pp.11-13. 
-Una dictadura militar sería tan funesta o más que la que venimos padeciendo, porque por mucho saber que supusiéramos en el Gobierno que formasen diez hombres con uniforme, ni su temperamento, ni su preparación en los múltiples y complejísimos problemas de España serían garantía suficiente para despejar el horizonte nacional. ${ }^{21}$.

Pese a lo expuesto en estas líneas, La Unión y su representada -la Unión Comercial- consideraron positivo el derrocamiento de la vieja política por un régimen interino de corte militar, *por lo menos para dar fe de la transformación del sistema político hasta ahora imperante, haciendo desembocar la situación en una nueva donde fueran *los órganos naturales de la sociedad española los que se encarguen de regirse. ${ }^{22}$.

Por otra parte, son conocidos los refrendos que recibió el golpe de estado por las organizaciones externas al sistema canovista. En Sevilla y para estos primeros días, los católicos, los mauristas y la Unión Comercial fueron los principales e incondicionales cimientos del embrionario régimen en la derecha del espectro político. Esos apoyos se derivaron de la identificación de propósitos que mantenían las promesas del Directorio con el ideario regeneracionista del conservadurismo español del momento ${ }^{23}$. De hecho, con respecto al área de la Administración Local y Provincial, la Dictadura prometía a llevar a la práctica los planteamientos de la corriente renovadora de la derecha destilada de la insatisfacción del sistema del turno: descuaje del caciquismo; descentralización administrativa; separación de política y administración.

Las Diputaciones no serían sustituídas hasta enero de 1924 (Real Decreto de 12 enero 1924), una vez renovados los Gobernadores Civiles (20 de septiembre de 1923), los Ayuntamientos (30 del mismo mes) y constituídos los delegados gubernativos (20 de octubre). Siendo la última pieza del sistema administrativo a poner a punto, las viejas Diputaciones sobrevivieron el tiempo suficiente como para sufrir desde su propio seno la sucesión implacable de cambios formales del Directorio militar en materia de Administración local. Así, los efectos del golpe fueron recibidos en la Diputación de Sevilla con una mezcla de adhesión y obligada admisión de los hechos consumados ${ }^{24}$. Sentir una u otra sensación dependió del encuadramiento político y de los planes de futuro que cada

(21) LA UNION, 15 de septiembre de 1923.

(22) Ibídem.

(23) Tusell Gómez, J. y Chacón Ortíz, D.: La reforma de la Administración local en España (1900-1936) (Madrid, Instituto de Estudios Administrativos, 1973).

(24) En otras Diputaciones se experimentaron similares sensaciones frente al golpe. Vid.: Lemus López, E.: Las Diputaciones de Badajoz y Câceres durante la Dictadura de Primo de Rivera (1923-1930), (Sevilla, T.D.I., 1990), ff.22-41 passim. 
diputado se hiciera. De inmediato la Diputación, bajo el ojo escrutador del nuevo Gobernador civil (general Perales) y el ejemplo de las drásticas medidas adoptadas por el Directorio con respecto a Ayuntamientos, se refugió en su esfera de gestión administrativa. Ello no le valió, sin embargo, para que pronto el Gobernador interviniera en los asuntos de la Diputación con ocasión de proveer la plaza de Depositario de los Fondos Provinciales que se encontraba vacante por el fallecimiento de su titular ${ }^{25}$. Mientras los diputados conservadores -con la oposición de los albistasconsideraban de imperiosa necesidad* la dotación de la mencionada vacante haciéndola recaer en un empleado de la Diputación, el Gobernador se negó a ello en virtud de la prohibición temporal de nombrar nuevos cargos ${ }^{26}$. Lo cierto es que, tras el debate sobre la cuestión de la Depositaría, que aun daría mucho que hablar, se escondía un clima de inquietud e incertidumbre por el futuro que dejaba entrever El Correo cuando comentaba el desarrollo de la primera sesión de octubre:

-Después de dos horas de deliberaciones se levantó la sesión, y hasta otro lunes, en que nos parece que volveremos a escuchar el mismo disco de las denuncias sobre el Hospital, de la provisión de la depositaría y de los eternos y soporíferos recursos de alzada. Eso si es que hay Diputación para otro lunes porque al paso que vamos... ni alcaldes de barrio nos van a quedar ${ }^{27}$.

Y es que como señalaba el órgano católico, era creencia generalizada en el animado y regenerador ambiente político de estos momentos la inminente sustitución de las Diputaciones Provinciales: ‘organismos estériles cuando no perniciosos, que deberán ser sustituídos por la Diputación regional, que solamente entiendan en cuestiones de Beneficencia, administrando las consignaciones que el Estado les dé, ${ }^{28}$. Sólo bajo esta convicción se entiende la súbita ruptura que, con sus dimisiones, protagonizarían los diputados sevillanos pertenecientes a la Unión Comercial, García Junco y Soler Navarro.

En carta publicada el 5 de octubre por el diario La Unión y dirigida al Gobernador Civil, los diputados mencionados presentaron sus renuncias alegando que habían representado a la Unión Comercial en la Comisión Provincial "con voz, pero sin voto*, extremo cierto pues no pertenecían a la Permanente. Mas dudoso, sin embargo, es que ese detalle fuera garantía de validez para justificar sus dimisiones, si descontamos el desacuerdo de que siempre habían hecho gala con respecto a la gestión de

(25) A.D.P.S. Actas Comisión Provincial, sesión de 24 de septiembre de 1923, f.275 r.

(26) Ibídem, sesión de 1 de octubre de 1923, ff.292r-293.

(27) EL CORREO DE ANDALUCIA, 2 de octubre de 1923.

(28) Declaraciones de Salcedo -Presidente de la Diputación de Madrid- tras su entrevista con Martínez Anido. Vid.:EL. CORREO DE ANDALUCIA, 5 de octubre de 1923. 
la beneficencia los diputados de la Unión Comercial, tal y como pretendieron argumentar:

.Por estas razones somos por completo ajenos y por consiguiente irresponsables moral y materialmente de la actuación pasada de la Diputación Provincial y de la actual en lo que con la organización y régimen de los establecimientos benéficos se refiere... ${ }^{29}$.

Pero, en realidad, este abandono de la Diputación respondía a razones de claro interés coyuntural que iban a llevar en pocos meses a la Unión Comercial, desde su maridaje con los conservadores, hacia un primer acercamiento a la Dictadura. La prueba de encuentra en las consideraciones de ambos diputados:

.Hacemos estas manifestaciones -prosiguen en su escrito al Gobernador- porque no queremos, cuando llegue el momento próximo de la disolución de las actuales Diputaciones provinciales... caer envueltos en el descrédito y censuras que más o menos rodean a estas Corporaciones y a los que de ellas formamos parte, y como pretendemos un régimen de excepción para nosotros, antes de salir echados como ineptos o malos administradores, y lo que es peor, acusados de faltas que no hemos cometido, presentamos a V.E. la renuncia de nuestros cargos de diputados provinciales. ${ }^{30}$.

Es obvio, por tanto, que estas dimisiones se encontraban encuadradas en la estrategia de la Unión Comercial de acercamiento al nuevo régimen haciendo olvido de sus pasados devaneos con la vieja política local que fue, paradójicamente, la que les llevó hasta la Diputación ${ }^{31}$. Lógicamente estas dimisiones recibieron contestación por los diputados conservadores desde la prensa y, justo al día siguiente, Sarasúa refrescó la memoria a los dimisionarios recordándoles que sus cargos fueron el resultado del concierto celebrado entre el propio presidente de la Unión Comercial, Juan María Moreno, y los viejos partidos. Continuaba su exposición inquiriéndoles Sarasúa sobre si creían "poseer la patente de aptitud, capacitación y moralidad" o si, por el contrario, "al correr de la pluma vertieron especies que no pensaron debidamente, no olvidándose de advertir a los diputados renunciantes -apuntándose Sarasúa su propia loa implícita de la Dictadura- que:

.... mal concepto han formado ustedes del Directorio militar, que seguramente no dirá, llegado el caso de la disolución de las

(29) LA UNION, 5 de octubre de 1923.

(30) Ibídem.

(31) Alvarez Rey, L.: .Organizaciones patronales durante la Dictadura de Primo de Rivera: la Unión Comercial Sevillana. en Revista de Historia Contemporánea, (Dpto. de Historia Moderna y Contemporánea, Univ. de Sevilla), núm.4, diciembre 1985, pp.173-174. 
Diputaciones, lo que ustedes temen, porque no entra en su programa inferir agravios.32.

No acabaría aquí el cruce epistolar entre los diputados unionistas y conservadores. Las réplicas y contrarréplicas entre unos y otros durarían algún tiempo y no harían más que desvelar las diferencias existentes entre el caduco conservadurismo canovista y la configuración de una nueva derecha nacida de las limitaciones del sistema del turno. Una nueva derecha que, dentro de su heterogeneidad, valoraba la Dictadura como la oportunidad regeneradora capaz de emprender, ineludiblemente, una reforma de la administración española en sus esferas local y provincial, verdaderos viveros del caciquismo hasta entonces.

A partir de la dimisión de los diputados de la Unión Comercial, la Diputación sevillana inició una cuenta atrás hacia su disolución mientras realizaba esfuerzos encaminados hacia su acomodación en la política del Directorio, aun por estas fechas muy indefinida en materia provincial. En ese sentido, es de destacar el estudio que encomendó la Corporación a los diputados Revilla y Tassara para que analizaran ulos propósitos que animan al Directorio militar sobre [la] transformación de las provincias en regiones [y] propongan a esta corporación lo que sea más conveniente a los intereses de la región andaluza ${ }^{33}$. Estos esfuerzos eran paralelos a los que, con carácter particular, realizarían también elementos de la vieja política sevillana ${ }^{34}$.

\section{El nombramiento de la primera Diputación de la Dictadura (enero 1924)}

Por Real Decreto de 12 de enero de 1924 fueron configuradas las nuevas Diputaciones, aun con arreglo a la Ley de 1882 . En la de Sevilla, la disposición fue acatada sin discusión explícita por parte de la Corporación saliente. Y ello a pesar de que los diputados sustituídos habían sido consustanciales con la política provincial durante lustros: quince de ellos habían sido diputados en dos o más ocasiones, destacando el caso de Juan Florindo que, según El Correo, lo venía siendo desde $1900^{35}$. Los nuevos diputados fueron nombrados por el Gobernador Perales en una tarea nada simple pues ....no era sencillo encontrar a personas de solvencia y prestigio sociales absolutamente desvinculadas con el mundo de la

(32) EL CORREO DE ANDALUCIA, 6 de octubre de 1923.

(33) A.D.P.S. Actas Comisión Provincial, sesión de 5 de noviembre de 1923, ff.340 r-341.

(34) Es el caso, por ejemplo, del jefe conservador Carlos Cañal que pronunció una serie de conferencias en el Ateneo sevillano propugnando la resurrección del proyecto maurista de reforma del régimen local de 1907, actos criticados por los católicos por concebirlos como un cambio táctico de actitud por parte del conservadurismo local. Vid.: EL CORREO DE ANDALUCIA, 18 de noviembre de 1923

(35) EL CORREO DE ANDALUCIA, 15 de enero de 1924. 
política. ${ }^{36}$. Bajo presiones ciertas sobre el Gobernador ${ }^{37}$, éste logró designar los 28 diputados sevillanos en una fórmula de nombramiento que, por cierto, no se correspondía con la esperada descentralización y autonomía administrativa prometida para los órganos locales que, de esta manera, seguían dependiendo de su superior jerárquico, en una especie de neocaciquismo soterrado ${ }^{38}$. La nueva Diputación fue constituída el 20 de enero con el siguiente perfil ${ }^{39}$ :

\section{Sevilla, primer distrito}

CARLOS GARCIA OVIEDO. Catedrático de Derecho Penal y técnico en Administración. Preside el Ateneo e integra la Unión Patriótica Ciudadana. Anteriormente no ha figurado en política.

JOSE RAOUL NOEL. Banquero y naviero. Vocal de la Cámara Oficial de Comercio, Industria y Navegación. No ha figurado en política.

JOSE MARIA LOPEZ CEPERO Y MURU. Abogado y propietario. Secretario del Colegio de Abogados. Ha sido concejal y presidente de la Liga Católica (sustituyendo a Rojas Marcos tras su muerte). Preside el Partido Social Popular (P.S.P.)

CARLOS SERRA Y PICKMAN (Marqués de San José de Serra). Abogado y propietario. Ha sido concejal. Presidente del Consejo de Administración de la Cartuja.

Sevilla, segundo distrito

VICENTE HERNANDEZ IRALA. Designado como Presidente del Colegio Médico de Sevilla.

RICARDO DE ROJAS Y SOLIS (Marqués de Tablantes). Propietario. No ha figurado en cargo político. Gentílhombre de la Cámara del Rey, grande de España y jefe del Somatén.

MANUEL FONTAN AMAT. Farmacéutico y propietario. Designado como miembro del Colegio de Farmacéuticos.

GUILLERMO VILCHES. Catedrático Auxiliar de la Facultad de Medicina. [Fue pronto sustituído por ANTONIO RODRIGUEZ PALACIOS, profesor veterinario y académico de número de la Real Academia de Medicinal.

Distrito Utrera-Marchena

DANIEL MEZQUITA MORENO. Catedrático de Anatomía.

FERNANDO GONZALEZ IBARRA. Propietario, hijo del ex-alcalde González Alvarez.

(36) Tusell Gómez, J.: La crisis del caciquismo andaluz (1923-1930), (Madrid, Cupsa, 1977), p.65. Cursiva del autor. Sobre las dificultades para encontrar diputados ver EL LIBERAL, .Con la linterna de Diógenes..., 19 de enero de 1924.

(37) EL LIBERAL, 18 de enero de 1924.

(38) Tusell Gómez, J.: La crisis... op. cit., p.65.

(39) A.D.P.S. Actas del Pleno Provincial, sesión de 20 de enero de 1924, ff.28-30r. 
JOAQUIN GIRALDEZ RIAROLA. Abogado y propietario. Registrador de la Propiedad.

JOSE LOPEZ DE SAGREDO (Marqués de Castellón). Abogado y propietario. Según $E l$ Correo se podía considerar un garante contra la vieja política, pero lo cierto fue que, aunque no figuró activamente en política, estaba emparentado con el senador conservador Agustín Ternero.

\section{Distrito Cazalla-Sanlúcar}

ANGEL CAMACHO BAÑOS. Abogado, auxiliar en la Universidad. Sin figurar en política llegó a ser un hombre clave en la Diputación.

FEDERICO CASTEJON MAR'TINEZ DE ARIZALA. Abogado y Catedrático de la Universidad Imeses más tarde sería sustituído por JUAN MARIA MORENO].

ENRIQUE GARCIA CASTRILLON. Teniente Coronel de Caballería retirado. GARCIA DE PORRES Y PORRES. Abogado y propietario.

\section{Distrito Osuna-Morón}

MANUEL GONZALEZ PAREJO. Comerciante de aceites y propietario. No ha figurado en política.

FELICIANO CANDAU PIZARRO. Rector de la Universidad hispalense. Ha sido ex-concejal.

FRANCISCO VILLALON GORDILLO. Abogado y propietario, ex-alcalde de Morón.

ENRIQUE TELLO GARCIA. Decano de la Facultad de Medicina y propietario. Ha figurado en política.

\section{Distrito Ecija-Estepa}

FRANCISCO CLAVERO RAMIREZ. Abogado, fabricante de harina y vicepresidente del Círculo Mercantíl.

DOMINGO PEREZ GONZALEZ. Abogado, comerciante y propietario. Ex-concejal y ex-teniente alcalde, recién llegado a la Unión Patriótica.

JUAN CASTRILLO DIAZ. Abogado y propietario. Ex-teniente alcalde en Ecija.

JOSE LASARTE MARTIN. Abogado y propietario.

\section{Distrito Carmona-Lora}

FRANCISCO GARCIA RODRIGUEZ. Obrero tipógrafo en la imprenta de Eulogio de las Heras. Representa a la clase trabajadora. Fundó la Sociedad de Constructores de Carros y del Sindicato de la Aguja. En 1921, fue presidente de la Sociedad de Obreros del Arte de Imprimir.

CAYETANO SANCHEZ PINEDA. Abogado y propietario. Secretario de la Academia de Bellas Artes. ha figurado en política, siendo tres veces concejal.

SABAS MARIN JIMENEZ. Banquero y ex-comerciante de tejidos en Carmona. LAUREANO MONTOTO Y GONZALEZ DE LA HOYUELA. Médico y propietario. 
A la vista de las propios datos biográficos expuestos se infieren ciertos cambios en la formación de la Diputación. Si antes en ésta tan sólo aparecían hombres políticos, ahora se conformó una Corporación de cierto corte técnico y administrativo, destacando -por su carácter singular- el nombramiento del diputado obrero Francisco García de acuerdo con la estrategia inicial de entendimiento del Directorio con la clase trabajadora. El nuevo sentido técnico que se pretendió conferir a la Diputación parece demostrarlo el nombramiento de médicos y abogados para la gestión de este organismo que, en su regeneración, debía reconducirse hacia los derroteros de su principal misión: el mantenimiento y sustento de los establecimientos de la Beneficencia. En este sentido, las propias palabras del Gobernador en el acto de toma de posesión de los diputados fueron bien elocuentes cuando realizó un llamamiento a los nuevos cargos para thacer una gran labor, pues cuantos la constituyen no tienen presiones ni influencias de Jefes de Partidos, y en su gestión no deben tener más norte ni más fin que proceder siempre con arreglo a los dictados de su conciencia ${ }^{40}$. Por su parte, la prensa más adicta a las posiciones de los nuevos diputados no ahorró palabras en sus manifestaciones de entusiasmo y optimismo en relación a los mismos, con aseveraciones del siguiente tenor:

*...se ha renovado, no la mitad, sino la totalidad de las Diputaciones, y se ha hecho todo en 24 horas, sin anuncios, ni candidaturas, ni jaranas y sin que nadie, en justicia, pueda decir que los diputados elegidos sean de peor calidad que los que antes se elegían, sino más bien todo lo contrario ${ }^{41}$.

Pero si bien es cierto que fueron nombrados elementos nuevos para constituir la Corporación y que ningún diputado sustituído fue repuesto (ni siquiera los dimisionarios de la Unión Comercial, hecho comprensible dentro del distanciamiento temporal de esta organización con el Directorio por estos momentos), es también cierto que algunos elementos exvinculados a la vieja política seguían presentes en la Diputación ${ }^{42}$. Al respecto, aparte de ex-concejales, ex-tenientes de alcalde y ex-alcaldes que figuran en la relación, consignemos la filiación borbollista de Mez-

(40) Ibídem, f.29.

(41) EL CORREO DE ANDALUCIA, 20 de enero de 1924.

(42) Hasta el momento, existía un consenso generalizado acerca de la persistencia de la vieja política en los cuadros de la Dictadura de Primo de Rivera. Tras lo expuesto en este breve trabajo, se entiende que esa interpretación merece ciertas matizaciones pues es constatable la irrupción de nuevas figuras -especialmente católicos- en el escenario político tras el golpe del 13 de septiembre. Recientemente, estas consideraciones han sido recogidas en: Gómez Navarro, Jose Luís: El Régimen de Primo de Rivera. Reyes, dictaduras y dictadores, (Madrid, Cátedra, 1991), especialmente páginas 250 a 260. 
quita o la pasada militancia en el liberalismo de Clavero y Palacios. Como mínimo, un porcentaje próximo al $40 \%$ de los nuevos diputados se había encontrado vinculado con los partidos del turno, con independencia de aquellos que estaban emparentados con antiguos políticos y. aquellos otros que, debido a su posición económica, siempre habían disfrutado de gran influencia en las esferas de poder local (por ejemplo, Raoul Noel). Y es que aunque las intenciones de la Dictadura se concretaron en actuaciones muy diversas en función del marco provincial sobre el que se desarrollaron ${ }^{43}$, en Sevilla quedo claro desde el primer momento que la anhelada regeneración de la vida provincial iba a tener grandes limitaciones. Estas vendrían definidas, de una parte, por la propia incapacidad del Directorio para organizar totalmente la vida local y provincial sevillana sin contar con los viejos elementos que la habían controlado políticamente hasta entonces y, de otra, por la progresiva conversión en nuevos cacicatos -que servirían de apoyo al propio régimen- de las fuerzas emergentes en el panorama político sevillano del resto de la década de los veinte. Incluída, por supuesto, la Unión Patriótica.

(43) Tusell Gơmez, J.: La crisis... op. cit., p.55. 